Journal of Engineering and Applied Sciences 15 (6): 1346-1350, 2020

ISSN: 1816-949X

(C) Medwell Journals, 2020

\title{
Block Matching of Motion Estimation by using New Technique "Quintet Search Algorithm"
}

\author{
Salah A. Salman, Salah S. Mustafa and Saeed A. Gathban \\ Department of Computer Science, \\ College of Computer Science and Information Technology, University of Anbar, Ramadi, Iraq
}

\begin{abstract}
In digital video communication it is not sensible and practical to store the total digital video without handling because of the many problems and constraints in the process of storage and the transmitter, so, the method of processing called a video compression. In video compression, one of the most important techniques that contribute to getting compression ratio and speed in performance is the motion estimation. The motion estimation could be a method that determines the motion between two or more frames of video. In this study we have proposed a calculation of block matching for motion estimation named (Quintet search algorithm). This algorithm it is similar in terms of work (Three step search algorithm) with eight points but the proposed algorithm is five points to get the speed and compression ratio acceptable in less time and a really acceptable PSNR compared to the other ways.
\end{abstract}

Key words: Video compression, block matching, motion estimation, TSS, PSNR, communication

\section{INTRODUCTION}

In visual communications, the main objective is to obtain a high level processing speed and low computing time at the same time while not scarifying in picture quality (Ahmed and Aliesawi, 2013). Video compression has become important technology in a lot of operations in the storage or transmission, especially in the television broadcasting operations or closed conferences.

The video compression includes video coding to benefit from high correlation between sequential frames to enhancement coding efficiency which is often achieved by utilizing Motion Estimation (ME) and motion compensation methods. Motion estimation it is characterized as looking the better motion vector which is the relocation of the direction of the best similar block in previous frame for the block in current frame (Bachu and Chari, 2015). It is previously known that the video is sequential images be the difference between frame and another is almost non-existent where there is the highest level of repetition between frame.

In temporal redundancy the reduction of redundancy includes encoding between the previous frame and the current only this technique is called a Block Matching Algorithm (BMA). In this method we get good results in the speed and rate of compression has been named this way Quintet search algorithm.

Why motion estimation: In sequence images and the successive image often retain data between frames along motion trajectories. This is the similar to as saying that the scene content doesn't modification from frame to another.
To take advantage of the image information redundancy in image sequences there's a requirement to estimate motion within the image sequence.

Therefore, the use of motion estimation techniques depends on the threshold and the total of images to benefit from the process of repeating similar images (Baraskar et al., 2015).

\section{Block matching algorithms:}

- $\quad$ Exhaustive Search (ES)

- Three Step Search (TSS)

- $\quad$ New Three Step Search (NTSS)

- $\quad$ Simple and Efficient Search (SES)

- $\quad$ Four Step Search (4SS)

- Diamond Search (DS)

Block matching motion estimation: The principal purpose regarding block matching and motion estimation is that there's high correlation between every pixel and its neighbors. So, the purpose, distribution a motion vector to a block of pixels more beneficial than to single pixel (Bhaskaran and Konstantinides, 1995; Choudhury and Saikia, 2014). In the block matching motion estimation operation, a frame is metameric into $n \times n$ blocks. Then for the biggest motion displacement of 'p' pixels per frame; this block is matched with a corresponding block within the previous frame in same coordinates. The most effective match aboard in matching principle provides the displacement. Always have a choice affects the output results and PSNR (Lins et al., 2011). In the block matching motion estimation, every current frame is segmented into do not overlap square blocks of size $\mathrm{N} \times \mathrm{N}$

Corresponding Author: Saeed A. Gathban, Department of Computer Science,

College of Computer Science and Information Technology, University of Anbar, Iraq 


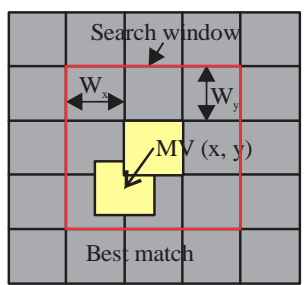

Reference frame

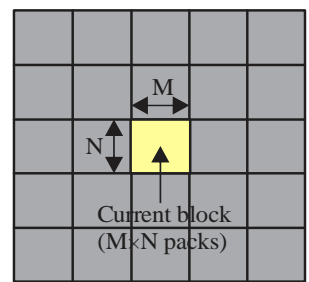

Current frame

2D-BM

Fig. 1: Dividing current frames into no overlapping blocks

as shown in Fig. 1. Considering a block within the current frame, known as current block, a search window of $\mathrm{N} \times \mathrm{M}$ blocks is defined around its corresponding location in reference frame, this is the previous frame. Then the algorithm tries to search out the best match for the current block within the search window in the reference frame (Moschetti et al., 2003; Moshe and Hel-Or, 2009).

The main objective and important of the block matching motion estimation algorithm is to work out the comparison between the pictures. The amount of similarity between the values in the image based the block matching operation.

Many types of parameters from block matching has been working out like Mean Square Error (MSE) is Meant the Absolute the Differences (MAD) and Peak Signals to Noise Ratio (PSNR) as represented in Eq. 1-3 (Sudhakar, 2014):

$$
\begin{gathered}
\text { MAD }=\frac{1}{N^{2}} \sum_{i=0}^{N-1} \sum_{j=0}^{N-1}\left|C_{i j}-R_{i j}\right| \\
M S E=\frac{1}{N^{2}} \sum_{i=0}^{N-1} \sum_{j=0}^{N-1}\left(C_{i j}-R_{i j}\right)^{2} \\
\text { PSNR }=10 \log _{10}\left[\frac{(255)^{2}}{M E S}\right]
\end{gathered}
$$

where, Mean Absolute Difference (MAD) and Mean Squared Error (MSE) tools measures of distortion or difference among the original frame and reconstructed frame. While Peak Signal to Noise Ratio (PSNR) measure image quality calculation depends on the Mean Squared Error (MSE) of among an original and an impaired video frame or image.

The proposed (quintet search algorithm): In this study proposes new algorithm are similar in terms of work three step search algorithm. It consists of five points at the beginning of research with emphasis on the coverage of the search window be the basis of the work of the
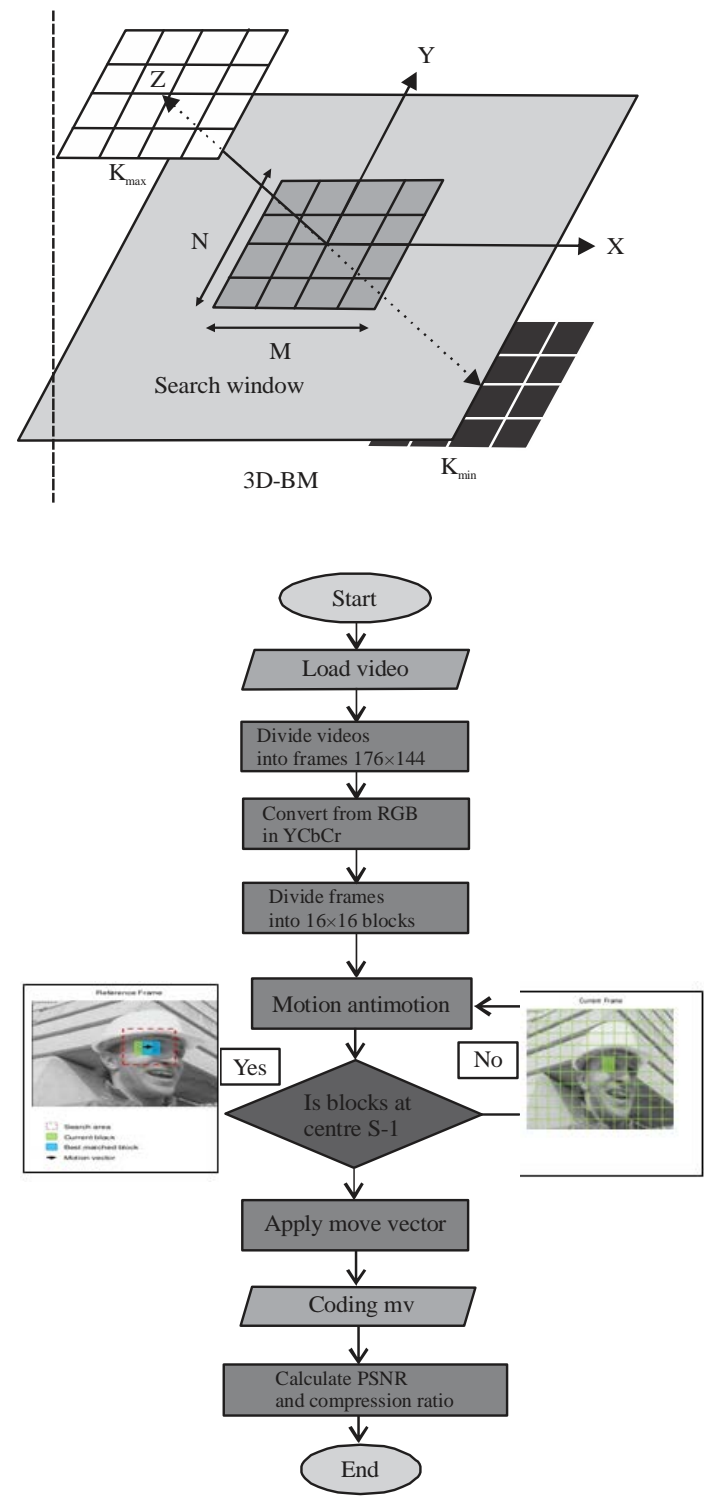

Fig. 2: The proposed algorithm for search and matching

Table 1: Input and output work scheme

\begin{tabular}{llll}
\hline No frame & 1 & $1-20$ & Output \\
\hline Size. Foreman & $48.5 \mathrm{~KB}$ & $970 \mathrm{~KB}$ & $32.4 \mathrm{~KB}$ \\
Type & $\mathrm{B} \mathrm{MP}$ & $\mathrm{B}$ MP & Video \\
Dimension & $177 \times 144$ & $177 \times 144$ & $177 \times 144$ \\
\hline
\end{tabular}

algorithm is to rely on motion estimation techniques. The main goal is important in this research is to reduce the points of research and get acceptable results in the video compression process, PSNR and reduce the time spent on the search process thus, eliminating the computational complexity. Table 1 that explains the work scheme.

Figure 2 it represents the steps of algorithm work from splitting the video into a frame to calculate compression ratio and image quality. Where the search for the match between an current frames and a previous 


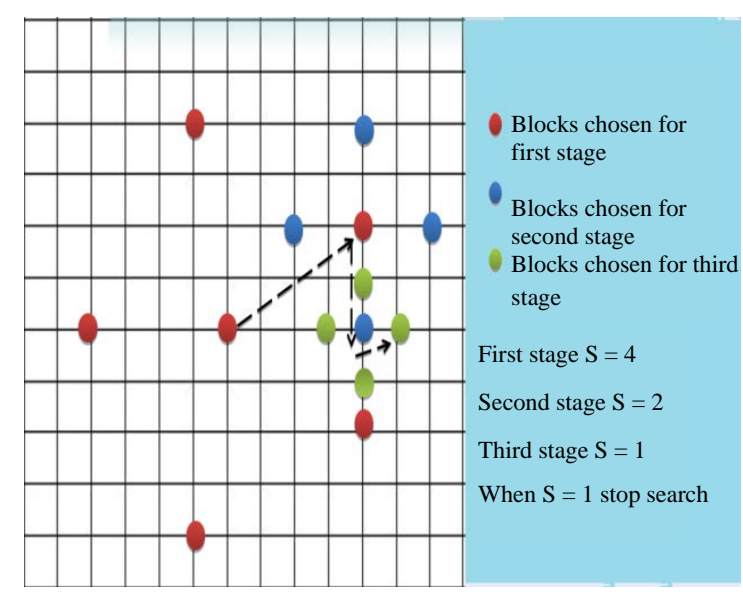

Fig. 3: Quintet search algorithm

frame. The split frame size to $16 \times 16$ is more efficient in the video compression process of the P-frame while I-frame will be $8 \times 8$ for higher efficiency.

The size of the frame used $(176 \times 144)$ that represents video conference applications. The search process is then performed according to the proposed algorithm in case there is a match (Move vector) the part is selected and the compression process is performed instead of pressing the entire frame. The compression ratio and the frame quality are calculated either in the absence of any match. The value of $S=1$ sends the frame to the compression part of I-frame. The process continues until the entire video frame is complete where the compression rate and frame quality are calculated according to the proposed search algorithm.

\section{MATERIALS AND METHODS}

Method and distribute the search points for the proposed algorithm: The proposed block matching algorithm works on the P-frame only the first stage is to open search window and distributing five initial positions across the $\mathrm{X}$ and $\mathrm{Y}$ axes $( \pm 4 \mathrm{X}, \pm 4 \mathrm{Y})$. Then the current block is shifted and compared to each block of the initial positions be less valuable than the five sites are MAD.

If this value is less than the threshold then this block is found: store a bit 1 as an indicator and 8 bits as motion vector. Motion vector usually be in a new location and is matching between the previous frame and the current frame the existence of the value of the threshold if less than the threshold be moving the second stage the minimum MAD value is considered the center and 4 points are distributed this time $( \pm 2 \mathrm{X}, \pm 2 \mathrm{Y})$.

The same steps are repeated and the same way of working with respect to the third stage after matching four points here and be the difference in size $( \pm 1 \mathrm{X}, \pm 1 \mathrm{Y})$, each stage $\mathrm{S} / 2$. Finally, in the absence of a match between the previous frame and the current in the third stage sites is discontinued $S=1$ using the I-frame compression steps.

Figure 3 represents the search process and the corresponding terms are split frame to micro block $16 \times 16$ and a comparison between the previous frame and the current account and the degree of difference a mong them this difference is called the move victor. Then, the compression of various processes and get the compression ratio, PSNR in the absence of match stop the search process this (I. frame). It sends the initial stage its compression.

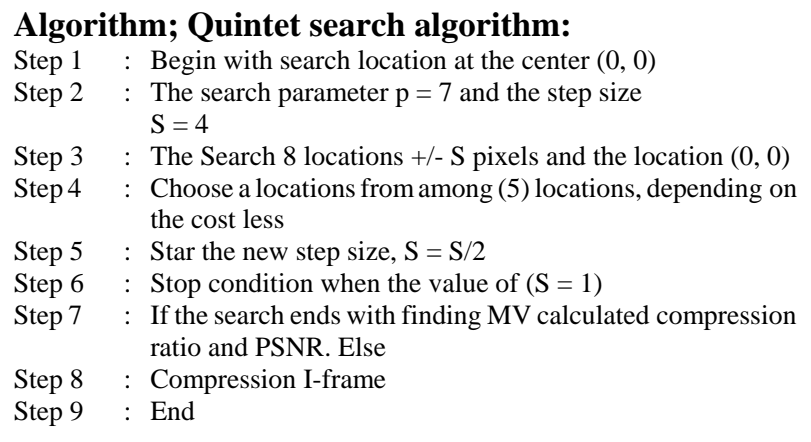

\section{RESULTS AND DISCUSSION}

In this research was conducted test to several videos which represents a clear image with a fixed background to get good results in the compression process and also PNSR. There are many factors that affect a direct impact on the results gained such as compression technique used, the lighting, the background, image quality, the number of images and the threshold which appeared in the results clearly.

Two videos were selected which represent record results that showed more quality results than manually recorded videos due to the quality of recording techniques. As show in Fig. 4.

Table 1 show the comparison with the most common algorithms with the proposed algorithm where the results showed the efficiency of the proposed algorithm in terms of pressure ratio and frame quality. Despite the great convergence of results, the proposed algorithm outperforms the quality of the frame.

This table represents the value of the average in general without knowing the technical details in terms of threshold or Gops and even the specifications of the computer. Table 2 represents the exact details of algorithm work with the selection of the value of the threshold plus the value of the set of images that were selected frame 10 and the calculation of the compression rate and frame quality and the results were good and acceptable. As in the Table 2. 
Table 2: Comparison with common algorithms parameter $=7$, size $16 \times 16$

\begin{tabular}{lcc}
\hline Name of algorithm & CR & PSNR \\
\hline FS & 29.078 & 32.119 \\
TSS & 30.261 & 30.717 \\
SES & 30.001 & 25.971 \\
NTSS & 30.125 & 27.020 \\
FSS & 29.225 & 31.345 \\
Quintet search & 30.145 & 32.535 \\
\hline
\end{tabular}

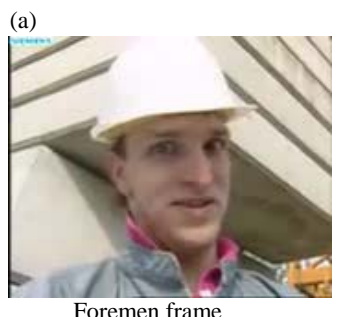

(c)

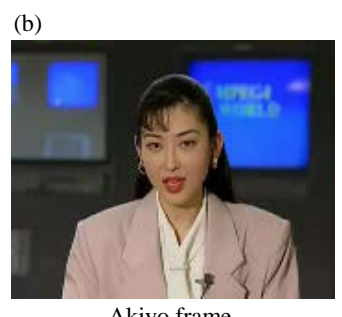

Akiyo frame

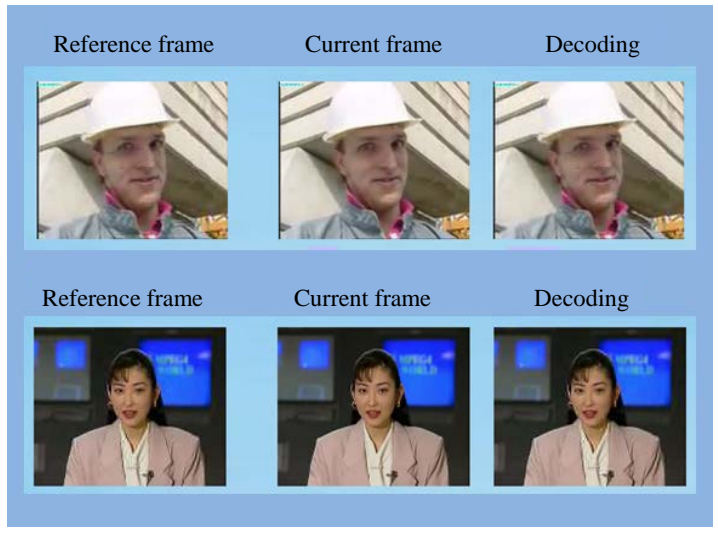

Fig. 4(a-c): Implementation and testing of the two video models

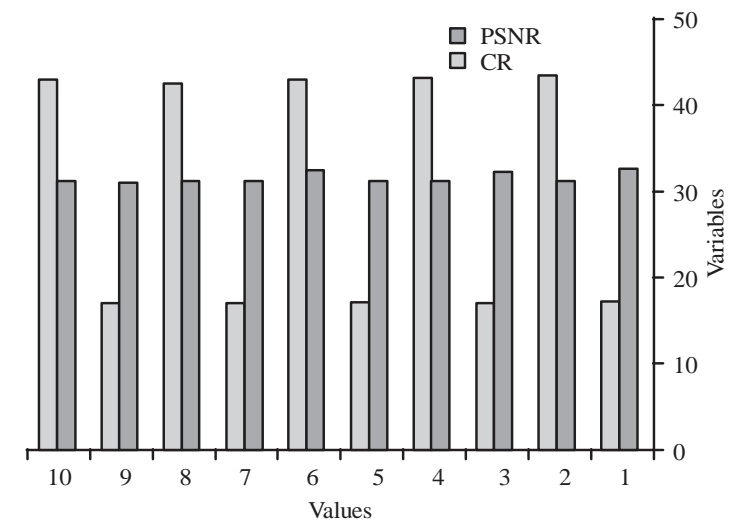

Fig. 5: The linear relationship between CR and PSNR

From Table 3, we conclude that the types of relationship are inverse between the compression ratio and the quality of the frame where the higher the compression the lower the quality coefficient of the image and vice versa. Figure 5 shows the relationship between CR and PSNR.
Table 3: Quintet search algorithm CR amd PSNR with time processing

\begin{tabular}{llllr}
\hline & GOP $=5$, Threshold $=2$ & Time & \\
& - & & \\
Frame No. $r$ & CR & PSNR & Coding & Decoding \\
\hline 1 & $1: 17.22$ & 32.8 & 00.4512 & 00.35650 \\
2 & $1: 43.57$ & 31.37 & 00.2132 & 00.19570 \\
3 & $1: 17.2$ & 32.32 & 00.1623 & 00.19090 \\
4 & $1: 43.28$ & 31.3 & 00.1961 & 00.17410 \\
5 & $1: 17.16$ & 31.27 & 00.2038 & 00.18580 \\
6 & $1: 43$ & 32.66 & 00.3886 & 00.34170 \\
7 & $1: 17.17$ & 31.2 & 00.1670 & 00.17880 \\
8 & $1: 42.62$ & 31.14 & 00.1613 & 00.16420 \\
9 & $1: 17.19$ & 31.11 & 00.1763 & 00.16740 \\
10 & $1: 43.04$ & 31.18 & 00.1562 & 00.16050 \\
Average & 30.145 & 32.535 & 0.2276 & 0.21156 \\
\hline
\end{tabular}

\section{CONCLUSION}

In this study, the focus on improving the rate of compression and quality of the frame in addition to the reduction of computations is an important factor in increasing the speed of processing where this algorithm proposed for search and matching is derived from the most common algorithms and efficiency (Three Step Search algorithm) with the reduction and redistribution of the research points where the proposed algorithm showed the efficiency of compression and processing speed in addition to the quality of the frame. From this we conclude that the lower the search points the faster the speed. Also, the relation between the compression rate and the quality coefficient inverse as the ratio of the pressure of the frame decreases in quality and vice versa.

\section{REFERENCES}

Ahmed, M.S. and S. Aliesawi, 2013. Efficient transform-based joint carrier offset and channel estimator for T-OFDM system. Proceedings of the IEEE International Conference on Electrical, Communication, Computer, Power and Control Engineering(ICECCPCE13), December 17-18, 2013, IEEE, Mosul, Iraq, pp: 204-207.

Bachu, S. and K.M. Chari, 2015. A review on motion estimation in video compression. Proceedings of the IEEE International Conference on Signal Processing and Communication Engineering Systems (SPACES), January 2-3, 2015, IEEE, Guntur, India, ISBN:978-1-4799-6110-8, pp: 250-256.

Baraskar, T., V.R. Mankar and R. Jain, 2015. Survey on block based pattern search techniques for motion estimation. Proceedings of the IEEE International Conference on Applied and Theoretical Computing and Communication Technology (ICATCCT), October 29-31, 2015, IEEE, Davangere, India, ISBN:978-1-4673-9223-5, pp: 513-518.

Bhaskaran, V. and K. Konstantinides, 1995. Image and Video Compression Standards: Algorithms and Architectures. Kluwer Academic Publishers, Boston, USA., ISBN-13: 9780792395911, Pages: 369. 
Choudhury, H.A. and M. Saikia, 2014. Survey on block matching algorithms for motion estimation. Proceedings of the IEEE International Conference on Communications and Signal Processing (ICCSP), April 3-5, 2014, IEEE, Melmaruvathur, India, ISBN:978-1-4799-3357-0, pp: 036-040.

Lins, R., E. Lima and S. Melo, 2011. Low discrepancy sequences applied in block matching motion estimation algorithms. Proceedings of the 24th IEEE Conference on Graphics, Patterns and Images (SIBGRAPI), August 28-31, 2011, IEEE, Maceio, Brazil, ISBN:978-1-4577-1674-4, pp: 205-210.
Moschetti, F., M. Kunt and E. Debes, 2003. A statical adaptive block matching motion estimation. IEEE. Trans. Circuits Syst. Video Technol., 13: 417-431.

Moshe, Y. and H. Hel-Or, 2009. Video block motion estimation based on gray-code kernels. IEEE. Trans. Image Process., 18: 2243-2254.

Sudhakar, 2014. Evaluation and comparison of different fast block matching algorithms using motion estimation. Proceedings of the International IEEE Conference on Science, Engineering and Management Research, November 27-29, 2014, IEEE, Chennai, India, ISBN:9781479974412, pp: $745-745$. 